\title{
CONVOLUTION EQUIVALENCE AND INFINITE DIVISIBILITY: CORRECTIONS AND COROLLARIES
}

\author{
ANTHONY G. PAKES, ${ }^{*}$ University of Western Australia
}

\begin{abstract}
Corrections are made to formulations and proofs of some theorems about convolution equivalence closure for random sum distributions. These arise because of the falsity of a much used asymptotic equivalence lemma, and they impinge on the convolution equivalence closure theorem for general infinitely divisible laws.
\end{abstract}

Keywords: Subexponential distributions; convolution equivalence; infinite divisibility; random sums; tail equivalence

2000 Mathematics Subject Classification: Primary 60E07; 60F99

\section{Introduction}

The status of several closure theorems for convolution equivalent distributions has recently been questioned. This is because their proofs rely, directly or indirectly, on Lemma 2.1(iv) of Cline (1987). Shimura and Watanabe (2005) exhibit a counterexample to Cline's lemma that, in particular, affects part of the principal results obtained by Pakes (2004). The specific issue is whether convolution equivalence of a random sum distribution implies the convolution equivalence of the summand distribution. The proofs of the best existing results asserting that this is the case are incomplete as a result of the problem with Cline's lemma.

We discuss Cline's lemma in Section 2 and the random sum problem in Section 3. Theorem 3.1 below is a slightly weaker version of Theorem 5.1 of Pakes (2004) which preserves its three-way equivalence for random sum distributions. In a recent independent study by Wang et al. (2006), it was shown that if the compounding probabilities $p_{n}$ decrease sufficiently fast then the side condition (a) of our Theorem 3.2 holds. Theorem 1.2 of Wang et al. (2006), which corresponds to Corollary 2.14(i) of Cline (1987), embraces Poisson compounding and hence it can replace that part of the proof of Theorem 3.1 of Pakes (2004, p. 416) (reproduced as Theorem 3.1 below) which attempted to show that (c) in his Theorem 3.1 implies (a). Precisely, this implication asserts that if an infinitely divisible law is convolution equivalent then so is $J(x)=v[1, x) / v[1, \infty)$, where $v$ is the Lévy measure. Lemma 3.2, which corresponds to Corollary 2.14(ii) of Cline (1987), achieves the same end by altering some details of an argument in Shimura and Watanabe (2005).

The condition imposed on the compounding distribution by Wang et al. (2006) also embraces geometric compounding. In Section 4 we give a different and simpler proof for this case and show that it extends, using Theorem 3.3, to negative binomial and log-series compounds. Finally, for completeness, in Section 5 we prove closure for convolution roots in the two-sided case.

Received 16 November 2005; revision received $29 \overline{\text { November } 2006 .}$

* Postal address: Department of Mathematics and Statistics, University of Western Australia, 35 Stirling Highway, Crawley, WA 6009, Australia. Email address: pakes@maths.uwa.edu.au 


\section{Cline's lemma}

If $A_{i}: \mathbb{R} \mapsto \mathbb{R}(i=1, \ldots, 4)$ are positive on the interval $\left[x^{\prime}, \infty\right)$ and if $c \geq 0$, we write $A_{1}(x) \sim c A_{2}(x)$ if $\lim _{x \rightarrow \infty} A_{1}(x) / A_{2}(x) \rightarrow c$ and $A_{1}=o\left(A_{2}\right)$ if $c=0$; and we write $A_{1} \prec c A_{2}$ if $\lim \sup _{x \rightarrow \infty} A_{1}(x) / A_{2}(x) \leq c$, and $A_{1}=O\left(A_{2}\right)$ if $A_{1} \prec c A_{2}$ for some positive $c$.

Cline's lemma states that if the $A_{i}$ satisfy

$$
A_{1} \prec A_{3} \quad \text { and } \quad A_{2} \prec A_{4},
$$

and

$$
A_{1}+A_{2} \sim A_{3}+A_{4}
$$

then one of the following outcomes must occur:

$$
\left\{\begin{array}{lll}
A_{1} \sim A_{3} & \text { and } & A_{2} \sim A_{4}, \\
A_{1} \sim A_{3} & \text { and } & A_{4}=o\left(A_{2}\right), \\
A_{2} \sim A_{4} & \text { and } & A_{3}=o\left(A_{2}\right) .
\end{array}\right.
$$

Cline (1987) asserts that the proof is straightforward! The assertion is valid in the sense that any one, two, or all three of the outcomes (2.3) can occur along a subsequence if (2.1) and (2.2) hold. This is the point of the Shimura-Watanabe counterexample. However, the impact of this counterexample is lessened by the fact that the functions $A_{i}$ which Shimura and Watanabe (2005) use are not monotonic, whereas in all uses of Cline's lemma the $A_{i}$ are tail functions, and there is usually a lot of additional structure. The fallacy in the various uses of Cline's lemma is to infer the first outcome in (2.3) without eliminating the possibility that the others may hold along a subsequence.

The following lemma extracts the most that is possible from Cline's hypotheses.

Lemma 2.1. (i) Suppose that (2.1) holds. Condition (2.2) holds if and only if

$$
A_{3}-A_{1}=o\left(A_{3}+A_{4}\right) \text { and } A_{4}-A_{2}=o\left(A_{3}+A_{4}\right) .
$$

(ii) If (2.1) and (2.2) hold, then

$$
A_{1} \sim A_{3} \text { provided } A_{4}=O\left(A_{3}\right)
$$

and

$$
A_{2} \sim A_{4} \text { provided } A_{3}=O\left(A_{4}\right)
$$

Proof. (i) As

$$
1-\frac{A_{1}+A_{2}}{A_{3}+A_{4}}=\frac{\left(A_{3}-A_{1}\right)+\left(A_{4}-A_{2}\right)}{A_{3}+A_{4}},
$$

we see that (2.4) implies (2.2). Suppose that (2.2) holds. Assumption (2.1) implies that if $\varepsilon>0$ then there exists $x(\varepsilon)$ such that

$$
A_{3}(x)-A_{1}(x) \geq-\varepsilon A_{3}(x) \quad \text { and } \quad A_{4}(x)-A_{2}(x) \geq-\varepsilon A_{4}(x)
$$

if $x \geq x(\varepsilon)$. Hence, if $x>x(\varepsilon)$, the first equation of (2.7) yields

$$
\liminf _{x \rightarrow \infty} \frac{A_{3}-A_{1}}{A_{3}+A_{4}} \geq 0 .
$$


On the other hand, the second equation of (2.7) implies that

$$
1-\frac{A_{1}+A_{2}}{A_{3}+A_{4}} \geq \frac{A_{3}-A_{1}}{A_{3}+A_{4}}-\frac{\varepsilon A_{4}}{A_{3}+A_{4}} \geq \frac{A_{3}-A_{1}}{A_{3}+A_{4}}-\varepsilon,
$$

so

$$
0 \geq \limsup _{x \rightarrow \infty} \frac{A_{3}-A_{1}}{A_{3}+A_{4}} \geq-\varepsilon,
$$

and hence the first member of (2.4) follows. The second member of (2.4) follows in similar manner.

(ii) If (2.1) and (2.2) hold, then the first member of (2.4) implies that

$$
\frac{A_{3}-A_{1}}{A_{3}+A_{4}}=\frac{1-A_{1} / A_{3}}{1+A_{4} / A_{3}} \rightarrow 0
$$

and hence the numerator tends to zero provided $A_{4} / A_{3}$ is bounded. This implies (2.5), and similarly for (2.6).

\section{Random sums and convolution equivalence}

We consider a distribution function $G(x)<1$ for all real $x$, and denote its tail or survivor function by $\bar{G}=1-G$ and its convolution powers by $G^{* n}, n=1,2, \ldots$ We say that $G$ has an exponential tail with rate $\gamma \geq 0$, written $G \in \mathcal{L}_{\gamma}$, if

$$
\lim _{x \rightarrow \infty} \frac{\bar{G}(x-y)}{\bar{G}(x)}=\mathrm{e}^{\gamma y}, \quad-\infty<y<\infty,
$$

and we say that $G$ is convolution equivalent, written $G \in \mathcal{\gamma}_{\gamma}$, if $G \in \mathcal{L}_{\gamma}$ and the following limit exists:

$$
2 M:=\lim _{x \rightarrow \infty} \frac{\overline{G^{* 2}}(x)}{\bar{G}(x)}<\infty .
$$

It is known that $M=M_{G}:=\int_{-\infty}^{\infty} \mathrm{e}^{\gamma x} \mathrm{~d} G(x)$. Pakes (2004, Corollary 2.1(iii)) proves this by extension from the one-sided case. Several authors have offered proofs for the one-sided case, and with differing errors. Rogozin (2000) deals with the case $\gamma=0$, and he asserts that the case $\gamma>0$ was earlier treated by himself and M. S. Sgibnev using Banach algebra techniques. Foss and Korshunov (2007, Section 8) cite Rogozin and Sgibnev (1999) for this case, they offer remarks on the earlier literature, and their Theorems 3 and 7, which are relevant to this topic, have 'elementary' proofs.

Let $\left(p_{n}: n=0,1 \ldots\right)$ be a probability mass function with $p_{0}<1$ and let

$$
F=\sum_{n \geq 0} p_{n} G^{* n}
$$

Then $F$ is the distribution function of the random sum $\sum_{j=1}^{\mathcal{N}} Y_{j}$, where the summands are independent with distribution function $G$ and independent of $\mathcal{N}$, where $\mathrm{P}(\mathcal{N}=n)=p_{n}$. Theorem 5.1 of Pakes (2004) can be re-stated as follows. 
Theorem 3.1. (Equivalent to Theorem 5.1 of Pakes (2004).) Suppose that:

(i) $G \in \mathcal{L}_{\gamma}$ for some $\gamma \geq 0$ and $M_{G}<\infty$,

(ii) $\sum_{n} p_{n}\left(M_{G}+\varepsilon\right)^{n}<\infty$ for some $\varepsilon>0$.

Then the following assertions are equivalent:

(a) $G \in \wp_{\gamma}$;

(b)

$$
\lim _{x \rightarrow \infty} \frac{\bar{F}(x)}{\bar{G}(x)}=\sum_{n \geq 1} p_{n} n M_{G}^{n-1}
$$

(c) $F \in \varsigma_{\gamma}$ and $\bar{G} \neq o(\bar{F})$.

Note that Theorem 3.1(ii) is satisfied if $M_{G}<1$, which is a possible outcome in the two-sided case. Cline's lemma was used to support the argument that Theorem 3.1(c) implies Theorem 3.1(a). This is now in doubt, but the following weaker version is valid. Let $M_{F}=\int_{-\infty}^{\infty} \mathrm{e}^{\gamma x} \mathrm{~d} F(x)$.

Theorem 3.2. Suppose Theorem 3.1(i) and (ii) hold. Then Theorem 3.1(a) and (b) are equivalent, and each is equivalent to

(a) $F \in s_{\gamma}$ and $\bar{F}=O(\bar{G})$.

Proof. We need only show that Theorem 3.2(a) implies Theorem 3.1(a) because the remainder of the proof of Theorem 3.1 given in Pakes (2004) is valid. If $\gamma>0$ then $M_{F}=\gamma^{-1} \int_{0}^{\infty} \mathrm{e}^{\gamma x} \bar{F}(x) \mathrm{d} x$, so Theorem 3.1(i) and $\bar{F}=O(\bar{G})$ imply that $M_{F}<\infty$. Let $A_{1}=2 M_{F} M_{G}^{2} \bar{F}, A_{2}=2 M_{G} M_{F}^{2} \bar{G}, A_{3}=M_{G}^{2} \overline{F^{* 2}}$, and $A_{4}=M_{F}^{2} \overline{G^{* 2}}$. Pakes (2004) shows that if $F \in \delta_{\gamma}$ then (2.2) holds and, of course, $A_{1} \sim A_{3}$. The second part of Theorem 3.2(a) can be read as $A_{1}=O\left(A_{2}\right)$. It follows from Lemma 2.1(ii) of Cline (1987) that $A_{2} \sim A_{4}$, i.e. Theorem 3.1(a) holds.

In the one-sided case $G(0-)=0$, Cline (1987, Corollary 2.14) asserts sufficient conditions for the second part of Theorem 3.1(c) to hold, i.e. that $\liminf _{x \rightarrow \infty} \bar{F}(x) / \bar{G}(x)<\infty$. Our stronger condition merely replaces the lim inf with lim sup. Thus, the second part of Theorem 3.2(a) is only a small strengthening of that in Theorem 3.1(c). It is worth noting that Klüppelberg (1989) adds a density version of this restriction to her Theorem 3.2.

Lemma 3.4 below for the one-sided case $G(0-)=0$ asserts that the lim sup restriction in Theorem 3.2(a) can be replaced by a stronger restriction on $\left(p_{n}\right)$. Its proof relies on some preliminary results valid for the two-sided case, which are of interest in their own right. The following result appears as Lemma 5.6 of Pakes (2004), where $F$ and $G$ are arbitrary distribution functions.

Lemma 3.1. Suppose that $F, G \in \mathcal{L}_{\gamma}$ and $M_{F}, M_{G}<\infty$, and that $\bar{F} \prec a \bar{H}, \bar{G} \prec b \bar{H}$ where $H \in \delta_{\gamma}$ and $a, b>0$ are constants. Then

$$
\overline{F * G}(x)=\left(M_{G} \bar{F}(x)+M_{F} \bar{G}(x)\right)(1+o(1))+o(\bar{H}(x)), \quad(x \rightarrow \infty) .
$$

In addition, if $\bar{H}=O(\bar{F}+\bar{G})$ then

$$
\overline{F * G}(x) \sim M_{G} \bar{F}(x)+M_{F} \bar{G}(x) .
$$


The next lemma collects results for $F$ defined by (3.1) which are scattered through Pakes (2004) and proved here in greater detail. We need the following notation. For positive integer $N$ let $p_{n}(N)=\mathrm{P}\left(\mathcal{N}_{1}+\cdots+\mathcal{N}_{N}=n\right)$ where the $\mathcal{N}_{j} \mathrm{~s}$ are independent copies of $\mathcal{N}$, and let

$$
\kappa(N):=\left(\sum_{n \geq 0} p_{n}(N) n M_{G}^{n-1}\right)^{-1} .
$$

This reciprocal definition makes the following expressions tidier.

Lemma 3.2. Fix $N \in \mathbb{N}$, and assume that $G \in \mathcal{L}_{\gamma}, \kappa(N)>0$, and $F \in \mathcal{s}_{\gamma}$. Then

$$
\overline{G^{* N}} \prec \kappa(N) \overline{F^{* N}},
$$

and

$$
\overline{(F * G)^{* N}} \in s_{\gamma} .
$$

Proof. Suppose that $N=1$, and let

$$
\kappa=\kappa(1) \quad \text { and } \quad B_{n}(x)=\frac{\overline{G^{* n}}(x)}{\bar{G}(x)} .
$$

Lemma 5.4 of Pakes (2004) asserts that if $G \in \mathscr{L}_{\gamma}$ then

$$
\liminf _{x \rightarrow \infty} B_{n}(x) \geq n M_{G}^{n-1},
$$

and (3.4) follows. Observe that if $H=F$ in Lemma 3.1, then all its assumptions are satisfied and, in particular, (3.3) holds. It follows that $F * G \in \mathcal{L}_{\gamma}$ and, using (3.4), that

$$
\overline{F * G} \prec\left(M_{G}+\kappa M_{F}\right) \bar{F} .
$$

Hence, Lemma 3.1 can be invoked again with $F$ and $G$ in (3.2) replaced by $F * G$, giving

$$
\overline{(F * G)^{* 2}}=2 M_{F} M_{G} \overline{F * G}+o(\bar{F}) .
$$

As (3.3) implies that $\bar{F}=O(\overline{F * G})$, it follows that

$$
\overline{(F * G)^{* 2}}(x) \sim 2 M_{F} M_{G} \overline{F * G}(x),
$$

i.e. $\overline{F * G} \in \wp_{\gamma}$. Observing that $F^{* N}=\sum_{n \geq 0} p_{n}(N) G^{* n}$ and that the hypotheses imply that $G^{* N} \in \mathcal{L}_{\gamma}$ and $F^{* N} \in \mathcal{S}_{\gamma}$, we see that, for arbitrary $N$, (3.4) and (3.5) follow from the $N=1$ case.

Lemma 3.3. Suppose that $G \in \mathcal{L}_{\gamma}, F \in \mathcal{s}_{\gamma}$, and that $\kappa(N)>0$ for $N=1,2, \ldots$ Then

$$
N M_{F}^{N-1} M_{G}^{N} \bar{F}(x)+N M_{F}^{N} M_{G}^{N-1} \bar{G}(x) \sim M_{G}^{N} \overline{F^{* N}}(x)+M_{F}^{N} \overline{G^{* N}}(x) .
$$

Proof. For some $N \geq 1$ suppose that

$$
\overline{(F * G)^{* N}} \sim N M_{F}^{N-1} M_{G}^{N} \bar{F}+N M_{F}^{N} M_{G}^{N-1} \bar{G} .
$$


The case $N=1$ is just (3.3), and this is valid under our hypotheses. The assumptions of Lemma 3.2 are satisfied, so (3.5) implies that there are positive constants $a_{N}$ such that $\overline{(F * G)^{* N}} \prec a_{N} \bar{H}$, where $H=F * G$. As $\bar{H}=O(\bar{F}+\bar{G})$, it follows, again from (3.3), that

$$
\overline{(F * G)^{*(N+1)}}=\overline{(F * G)^{* N} *(F * G)} \sim M_{F} M_{G} \overline{(F * G)^{* N}}+\left(M_{F} M_{G}\right)^{N} \overline{F * G} .
$$

Applying (3.7) and (3.3), respectively, it follows that (3.7) holds with $N$ replaced by $N+1$ whence, by induction, it holds for all $N$.

The hypotheses imply that $F^{* N} \in \S_{\gamma}$ and $G^{* N} \in \mathcal{L}_{\gamma}$ for all $N$. Taking $H=\overline{F^{* N}}$ in Lemma 3.1, the estimate (3.4) permits the use of (3.3) to obtain

$$
\overline{(F * G)^{* N}}=\overline{\left(F^{* N}\right) *\left(G^{* N}\right)} \sim M_{G}^{N} \overline{F^{* N}}+M_{F}^{N} \overline{G^{* N}} .
$$

Combining this with (3.7) yields (3.6).

In the remainder of this section only, we let $q=\sum_{n \geq 1} p_{n}$. We next prove our foreshadowed one-sided alternative to Theorem 3.2.

Lemma 3.4. Suppose Theorem 3.1(i) holds and that $G(0-)=0$. Then Theorem 3.1 $(a)$ and $(b)$ are equivalent, and each implies Theorem 3.2(a). Conversely, if

(a) $F \in \delta_{\gamma}$ and $\sum_{n \geq 1} p_{n}\left[\left(M_{F}+\delta\right) / q\right]^{n}<\infty$ for some $\delta>0$, then Theorem 3.1(a) holds.

Proof. We need to show only that Lemma 3.4(a) implies Theorem 3.1(a). The key step is following a line of argument in Shimura and Watanabe (2005, p. 454) to show, for some $N \geq 1$, that

$$
\bar{F}=O\left(\overline{G^{* N}}\right) .
$$

Our assumptions imply that $\left(\overline{G^{* n}}\right)$ is a nondecreasing sequence and hence $\bar{F}=\sum_{n \geq 1} p_{n} \overline{G^{* n}} \geq$ $q \bar{G}$, in other words, $1-q+q G \geq F$. Using the binomial theorem to expand the $n$-fold convolution power of this inequality leads to the (crude) bound $q^{n} \overline{G^{* n}} \leq \overline{F^{* n}}$, valid for $n \geq 1$. It follows that

$$
1=S:=\frac{\sum_{n \geq 1} p_{n} \overline{G^{* n}}}{\bar{F}} \leq \frac{\sum_{n \geq 1} p_{n} q^{-n} \overline{F^{* n}}}{\bar{F}} .
$$

As $F \in \delta_{\gamma}$, for each constant $\delta>0$ there is a positive constant $K$ such that $\overline{F^{* n}}(x) / \bar{F}(x) \leq$ $K\left(M_{F}+\delta\right)^{n}$, valid for all $x>0$ and $n$; see Chover et al. (1973). It follows from the bound assumption in Lemma 3.4(a) that the second sum in (3.9) converges uniformly in $x>0$. Consequently, for arbitrary $0<\varepsilon<1$, we can find $N$ so large that the first sum in (3.9), summed over $n>N$ for $S$, is uniformly bounded above by $\varepsilon$. The remaining finite sum for $S$ is bounded above by $Q_{N} \overline{G^{* N}} / \bar{F}$, where $Q_{N}=\sum_{n=1}^{N} p_{n}$, and (3.8) follows.

Next, we show that Lemma 3.4(a) implies that $\kappa(N)>0$. As the radii of convergence of the power series $h_{N}(s):=\sum_{n>0} p_{n}(N) s^{n}=h_{1}^{N}(s)$ coincide, it follows from Lemma 3.4(a) that $\sum_{n \geq 0} p_{n}(N)\left[\left(M_{F}+\delta\right) / q\right]^{\bar{n}}<\infty$. As $M_{G} \geq 1$, we have $M_{F}=\sum_{n \geq 0} p_{n} M_{G}^{n} \geq q M_{G}$, and it follows that $\sum_{n \geq 1} p_{n}(N)\left(M_{G}+\delta / q\right)^{n}<\infty$, whence $\kappa^{-1}(N)=\sum_{n \geq 0} p_{n}(N) n M_{G}^{n-1}<\infty$, as desired.

It follows from our hypotheses and the last step that (3.6) holds. We choose $N$ so that (3.8) is valid. Denoting the four terms in (3.6) by $A_{1}, A_{2}, A_{3}$, and $A_{4}$, respectively, we see that (2.2) holds. In addition, $A_{1} \sim A_{3}$ because $F \in \delta_{\gamma}$, and (3.8) asserts that $A_{1}=O\left(A_{4}\right)$, i.e. $A_{3}=O\left(A_{4}\right)$. It follows from Lemma 2.1 that $A_{2} \sim A_{4}$, i.e. Theorem 3.1(a) is true. 
Lemma 3.2 is valid if $\left(p_{n}\right)$ is a Poisson distribution and hence, the closure theorem for positive compound Poisson laws implicit in Cline (1987, p. 358), and stated explicitly as Theorem 5.2 of Goldie and Klüppelberg (1998) (and elsewhere), is valid as stated. This particular case can be used in place of Cline's Theorem 2.13 and Corollary 2.14, at the end of the proof of Theorem 3.1 in Pakes (2004, p. 416). Consequently, Theorem 3.1 of Pakes (2004) is valid as stated and, for completeness, we give it as follows.

Theorem 3.3. Suppose that $F$ is an infinitely divisible distribution function with Lévy measure $v(d x)$, and let

$$
J(x)=\frac{v([x, \infty))}{v([1, \infty))} .
$$

The following assertions are equivalent:

(a) $J \in \ell_{\gamma}$,

(b) $J \in \mathcal{L}_{\gamma}$ and

$$
\lim _{x \rightarrow \infty} \frac{\bar{F}(x)}{v(x, \infty)}=M_{F}<\infty,
$$

(c) $F \in \delta_{\gamma}$.

\section{Negative binomial mixtures}

Results mentioned by Shimura and Watanabe (2005) as uncertain include geometric compound distributions. In this section we rescue and generalize this to the negative binomial case $\phi_{\zeta}(s):=\sum p_{n} s^{n}=[(1-q) /(1-q s)]^{\zeta}$, where $0<q<1$ and $\zeta>0$, and we let $F_{\zeta}=\phi_{\zeta}(G)$, but drop the subscript $\zeta$ in the case $\zeta=1$. In addition, we consider the generalized log-series distribution with probability generating function $L_{\lambda}(s)=[\log (1-q s) / \log (1-q)]^{\lambda}$, and let $H_{\lambda}=L_{\lambda}(G)$, where $\lambda>0$.

Theorem 4.1. Suppose that $G \in \mathcal{L}_{\gamma}$ and $q M_{G}<1$. Then the following are equivalent:

(a) $G \in \varsigma_{\gamma}$;

(c)

$$
\begin{aligned}
& \lim _{x \rightarrow \infty} \frac{\bar{F}_{\zeta}(x)}{\bar{G}(x)}=\phi_{\zeta}\left(M_{G}\right) ; \\
& \lim _{x \rightarrow \infty} \frac{\bar{H}_{\lambda}(x)}{\bar{G}(x)}=L_{\lambda}\left(M_{G}\right) ;
\end{aligned}
$$

(d) $F_{\zeta} \in \&_{\gamma}$ for some (and hence all) $\zeta>0$;

(e) $H_{\lambda} \in \diamond_{\gamma}$ for some (and hence all) $\lambda>0$.

Proof. Our assumptions imply that Theorem 3.1(ii) holds. Theorem 3.2 asserts that Theorem 3.1(a), (b), and (c) are equivalent, and that Theorem 3.1(a) implies Theorem 3.2(a) and Lemma 3.4(a). Clearly, $F_{\zeta}$ is a one-dimensional distribution function of a Lévy process with time parameter $\zeta$, so it follows from Theorem 3.3 that $F_{\zeta} \in s_{\gamma}$ for all $\zeta>0$, or for no $\zeta$. Hence, Theorem 3.2(a) holds if and only if $F_{1} \in 8_{\gamma}$.

Assume Theorem 3.2(a) holds. For $\zeta=1$, the identity $\phi(s)=1-q+q s \phi(s)$ implies, via $\phi_{F}=1-q \phi_{G} \phi_{F}$, that $\bar{F}=q \overline{F * G}$. Taking $H=F$ in Lemma 3.1 and observing that $\bar{F}=O\left(M_{G} \bar{F}+M_{F} \bar{G}\right)$ we have

$$
\overline{F * G} \sim M_{G} \bar{F}+M_{F} \bar{G} .
$$


Consequently, $M_{F} \bar{G} / \bar{F} \rightarrow q^{-1}-M_{G}>0$. Thus, Theorem 3.1(b) holds and hence $G \in \delta_{\gamma}$, i.e. Theorem 3.2(a) is equivalent to Theorem 3.1(a).

The distribution functions $H_{\lambda}$ also comprise of one-dimensional distribution functions of a Lévy process, and hence Theorem 4.1(e) holds if and only if $H_{1} \in \delta_{\gamma}$. But $H_{1}$ is the distribution function of jumps in the compound Poisson representation of $F_{1}$, and so it follows from Theorem 3.3 that $H_{1} \in \wp_{\gamma}$ if and only if $F_{1} \in \wp_{\gamma}$.

A consequence of this result is that the proof of Corollary 3.3 and the results discussed in Section 4 of Klüppelberg (1989) remain valid.

\section{Closure for convolution roots}

One implication of Theorem 3.3 is that if one element of a continuously indexed convolution semigroup is in $\delta_{\gamma}$, then all elements are in $\delta_{\gamma}$. The corresponding result for ordinary convolution powers $G^{* n}$ is included here for completeness. Theorem 5.1(a) appears in the unpublished report of Willekens (1987), but the details of our proof differ somewhat from his proof, being closer to the argument used by Embrechts and Goldie (1982) for the one-sided case.

Recall that if $G \in \mathcal{L}_{\gamma}$ and $B_{n}(x):=\overline{G^{* n}}(x) / \bar{G}(x)$ then

$$
\liminf _{x \rightarrow \infty} B_{n}(x) \geq \lambda_{n}:=n M_{G}^{n-1}, \quad n=1,2, \ldots
$$

Theorem 5.1. Suppose that $G \in \mathcal{L}_{\gamma}$ and $M_{G}<\infty$.

(a) If there is an integer $N \geq 2$ such that $L_{N}:=\limsup _{x \rightarrow \infty} B_{N}(x) \leq \lambda_{N}$ then $G \in 8_{\gamma}$.

(b) If $G^{* N} \in s_{\gamma}$ then $G \in s_{\gamma}$.

Proof. (a) In view of (5.1) the hypothesis actually asserts that $L_{N}=\lambda_{N}$. First, we show that

$$
L_{n+1} \geq M_{G} L_{n}+M_{G}^{n}, \quad n \geq 1 .
$$

As $\overline{G^{*(n+1)}}(x)=\int_{-\infty}^{\infty} \overline{G^{* n}}(x-y) \mathrm{d} G(y)$ we have $B_{n+1}(x)=I_{n}(x)+J_{n}(x)$, where

$$
I_{n}(x)=B_{n}(x) \int_{-\infty}^{x^{\prime}} \frac{\overline{G^{* n}}(x-y)}{\overline{G^{* n}}(x)} \mathrm{d} G(y),
$$

and $x^{\prime}$ is a real constant. Observing that in the integral $x-y>x^{\prime}$ if $x>2 x^{\prime}$, and as $\mathcal{L}_{\gamma}$ is closed under convolution powers, for $\varepsilon>0$ we can choose $x^{\prime}>0$ so large that

$$
\frac{\overline{G^{* n}}(x-y)}{\overline{G^{* n}}(x)} \leq \frac{\overline{G^{* n}}\left(x-x^{\prime}\right)}{\overline{G^{* n}}(x)} \leq \mathrm{e}^{\gamma x^{\prime}}+\varepsilon .
$$

Hence, the dominated convergence theorem shows that the integral term converges as $x \rightarrow \infty$ and hence

$$
\limsup _{x \rightarrow \infty} I_{n}(x)=L_{n} \int_{-\infty}^{x^{\prime}} \mathrm{e}^{\gamma y} \mathrm{~d} G(y) .
$$

Using (5.1) of Pakes (2004), the complementary integral

$$
J_{n}(x)=\int_{-\infty}^{x-x^{\prime}} \frac{\bar{G}(x-y)}{\bar{G}(x)} \mathrm{d} G^{* n}(y)+\frac{\overline{G^{* n}}\left(x-x^{\prime}\right) \bar{G}\left(x^{\prime}\right)}{\bar{G}(x)} .
$$


Fatou's lemma shows that the lim inf of the integral is no smaller than $M_{G}^{n}$. As

$$
\lim \sup B_{n+1}(x) \geq \lim \sup I_{n}(x)+\lim \inf J_{n}(x),
$$

letting $x^{\prime} \rightarrow \infty$ yields (5.2). Taking $n=N-1$, as we may, we see that $\lambda_{N}=L_{N} \geq$ $M_{G} L_{N-1}+M_{G}^{N-1}$, implying that $L_{N-1} \leq \lambda_{N-1}$. It follows from (5.1) that $L_{N-1}=\lambda_{N-1}$, and iterating this argument yields $L_{2}=\lambda_{2}$, i.e. $G \in \delta_{\gamma}$.

Our proof for (b) follows the argument used by Embrechts and Goldie (1982, Theorem 2.10). A decomposition dual to that we used for (a) yields

$$
1=\left(B_{N}(x)\right)^{-1} \int_{-\infty}^{x^{\prime}} \frac{\bar{G}(x-y)}{\bar{G}(x)} \mathrm{d} G^{* N}(y)+g_{N}(x) .
$$

The dominated convergence theorem implies that, as $x \rightarrow \infty$, the first integral can be expressed as $(1+o(1)) \int_{-\infty}^{x^{\prime}} \mathrm{e}^{\gamma y} \mathrm{~d} G^{* N}(y)$. Identity (5.1) of Pakes (2004) can be used to obtain the decomposition $g_{N}(x)=K_{1}(x)+K_{2}(x)+K_{3}(x)$, where the $K_{i}$ s will be defined as we proceed.

First,

$$
K_{1}(x)=\int_{-\infty}^{x^{\prime}} \frac{\overline{G^{*(N-1)}}(x-y)}{\overline{G^{* N}}(x)} \mathrm{d} G(y) .
$$

We write the integrand as

$$
\frac{\overline{G^{*(N-1)}}(x-y)}{\overline{G^{* N(N-1)}}(x-y)} \cdot \frac{\overline{G^{* N(N-1)}}(x-y)}{\overline{G^{* N}}(x-y)} \cdot \frac{\overline{G^{* N}}(x-y)}{\overline{G^{* N}}(x)} .
$$

Equation (5.1) asserts that the limsup of the first factor in (5.4) is at most $\left[N M_{G}^{(N-1)^{2}}\right]^{-1}$. The convolution equivalence of $G^{* N}$ implies that the limit of the second factor in (5.4) is $(N-1) M_{G}^{N(N-2)}$, and the limit of the third factor is $\mathrm{e}^{\gamma y}$. As $x-y>x^{\prime}$ if $x>2 x^{\prime}$, it follows from the lim sup version of the dominated convergence theorem that

$$
\limsup _{x \rightarrow \infty} K_{1}(x) \leq\left(1-N^{-1}\right) M_{G}^{-1} \int_{-\infty}^{x^{\prime}} \mathrm{e}^{\gamma y} \mathrm{~d} G(y) .
$$

We define $K_{2}(x)$ as the integral in (5.3) but taken over [ $x^{\prime}, x-x^{\prime}$. If $x^{\prime}<y<x-x^{\prime}$ then $x-y \geq x^{\prime}$, so by taking $x^{\prime}$ sufficiently large we see, as above, that the first two factors in (5.4) together contribute a factor of at most $M_{G}^{-1}$. Hence, if $x>x^{\prime}$ then

$$
K_{2}(x) \leq M_{G}^{-1} \int_{x^{\prime}}^{x} \frac{\overline{G^{* N}}(x-y)}{\overline{G^{* N}}(x)} \mathrm{d} G(y) .
$$

If $X$ and $S$ are independent random variables having distribution function $G$ and $G^{* N}$, respectively, then the last integral equals

$$
\begin{aligned}
\mathrm{P}\left(x^{\prime}<X \leq x<S+X\right) \leq & \mathrm{P}(x<S \leq x+u) \\
& +\left[\mathrm{P}(S>x+u)+\mathrm{P}\left(x-x^{\prime}<S \leq x\right)\right] \mathrm{P}\left(x^{\prime}<X \leq x\right),
\end{aligned}
$$

where $u$ is a small positive number. It follows that $K_{2}(x)$ is of order

$$
1-\frac{\overline{G^{* N}}(x+u)}{\overline{G^{* N}}(x)}+\left[\overline{G^{* N}}(x+u)+\left(\overline{G^{* N}}\left(x-x^{\prime}\right)-\overline{G^{* N}}(x)\right)\right]\left(\frac{\bar{G}\left(x^{\prime}\right)-\bar{G}(x)}{\overline{G^{* N}}(x)}\right) .
$$


This quantity converges to $1-\mathrm{e}^{-\gamma u}+\left(\mathrm{e}^{-\gamma u}+\mathrm{e}^{\gamma x^{\prime}}-1\right) \bar{G}\left(x^{\prime}\right)$ as $x \rightarrow \infty$, and since $M_{G}<\infty$, this limit can be made arbitrarily small by choosing $u$ small and $x^{\prime}$ large.

Finally,

$$
K_{3}(x)=\frac{\bar{G}\left(x-x^{\prime}\right)}{\overline{G^{* N}}\left(x-x^{\prime}\right)} \cdot \frac{\overline{G^{* N}}\left(x-x^{\prime}\right)}{\overline{G^{* N}}(x)} \cdot \overline{G^{*(N-1)}}\left(x^{\prime}\right)=O\left(\mathrm{e}^{\gamma x^{\prime}} \overline{G^{*(N-1)}}\left(x^{\prime}\right)\right), \quad(x \rightarrow \infty)
$$

and this bound also can be made arbitrarily small by taking $x^{\prime}$ sufficiently large.

It follows from these estimates that for a given $\varepsilon>0$, we can choose $x^{\prime}$ so large and $u$ so small that if $x>2 x^{\prime}$ then

$$
1 \leq\left(B_{N}(x)\right)^{-1} \int_{-\infty}^{x^{\prime}} \mathrm{e}^{\gamma y} \mathrm{~d} G^{*(N-1)}(y)(1+o(1))+\left(1-N^{-1}\right) M_{G}^{-1} \int_{-\infty}^{x^{\prime}} \mathrm{e}^{\gamma y} \mathrm{~d} G(y)+\varepsilon,
$$

and hence, that

$$
1 \leq\left(\liminf _{x \rightarrow \infty}\left(B_{N}(x)\right)^{-1}\right) M_{G}^{N-1}+1-N^{-1}+\varepsilon .
$$

As $\varepsilon$ is arbitrary we conclude that $\lim _{\sup _{x \rightarrow \infty}} B_{N}(x) \leq N M_{G}^{N-1}$, and hence (a) implies that $G \in \diamond_{\gamma}$.

\section{Note added in proof}

Professor T. Watanabe has directed my attention to the fact that statement (c) in Theorem 3.3 above (and Theorem 3.1 of Pakes (2004)) should read:

(c) $J \in \mathcal{L}_{\gamma}$ and $F \in \delta_{\gamma}$.

The addition is required to ensure that Theorem 3.1(i) holds, and it seems to be necessary for any proof based on a random sum theorem that Theorem 3.3(c) implies Theorem 3.3(a). Watanabe (2007) proves that Theorem 3.3(c) implies Theorem 3.3(b) (and hence (a)) by using a clever adaptation of the first part of the proof of Theorem 4.2 of Embrechts and Goldie (1982). Theorem 3.3 as stated (i.e. Watanabe's version) is required for the assertions in the proof of Theorem 4.1 that, e.g. $F_{\zeta} \in \delta_{\gamma}$ for all $\zeta>0$, or for no $\zeta$. The proof can be recast to avoid any reference to infinite divisibility by using stronger random sum theorems, such as Theorem 1.2 of Wang et al. (2007). However, this results in a less elegant treatment.

\section{Acknowledgement}

I thank the referee for a thorough report which has helped me to improve the presentation of this paper.

\section{References}

Chover, J., Ney, P. And Wainger, S. (1973). Degeneracy properties of subcritical branching processes. Ann. Prob. 1, 663-673.

Cline, D. B. H. (1987). Convolutions of distributions with exponential and subexponential tails. J. Austral. Math. Soc. Ser. A 43, 347-365. (Correction: 48, (1990), 152-153.)

Embrechts, P. And Goldie, C. M. (1982). On convolution tails. Stoch. Process. Appl. 13, 263-278.

Foss, S. And Korshunov, D. (2007). Lower limits and equivalences for convolution tails. Ann. Prob.35, 366-383

Goldie, C. M. ANd Klüppelberg, C. (1998). Subexponential distributions. In A Practical Guide to Heavy Tails, eds R. Adler, R. Feldman and M. S. Taqqu, Birkhäuser, Boston, MA, pp. 435-459.

KLÜPPElberg, C. (1989). Subexponential distributions and characterizations of related classes. Prob. Theory Relat. Fields 82, 259-269. 
Pakes, A. G. (2004). Convolution equivalence and infinite divisibility. J. Appl. Prob. 41, 407-424.

Rogozin, B. A. (2000). On the constant in the definition of subexponential distributions. Theory Prob. Appl. 44, 409-412.

Rogozin, B. A. And Sgibnev, M. S. (1999). Strongly exponential distributions, and Banach algebras of measures. Siberian Math. J. 40, 963-971.

Shimura, T. And WatAnabe, T. (2005). Infinite divisibility and generalized subexponentiality. Bernoulli 11, 445-469.

WANG, Y., YANG, Y., WANG, K. AND Cheng, D. (2007). Some new equivalent conditions on asymptotics and local asymptotics for random sums and their applications. Insurance Math. Econom. 40, 256-266.

Watanabe, T. (2007). Convolution equivalence and distributions of random sums of IID. Submitted.

WiLleKens, E. (1987). Subexponentiality on the real line. Res. Rep., Katholieke Universiteit Leuven. 